\title{
Naming of Objects and Actions after Treatment with Phonological Components Analysis in Aphasia
}

\author{
Joana Kristensson, Charlotta Saldert \\ Institute of Neuroscience and Physiology, Division of Speech and Language Pathology, Sahlgrenska Academy, University of Gothenburg, \\ Gothenburg, Sweden
}

Objectives: To explore the effects of intensive treatment with phonological components analysis on naming of both objects and actions.

Methods: Phonological components analysis (PCA) was used to treat two individuals with moderate or severe aphasia after stroke. A single case study design with chronological time series analysis was employed to explore the effects of treatment for a total of up to 20 hours comprising the naming of self-chosen objects and actions. Changes in confrontation naming ability were assessed repeatedly. In addition, generalisation of training effects was examined in connected speech in a picture description task as well as in ratings of functional communication skills as perceived by the participants and their next of kin.

Results: An increase in correctly produced words for treated items with some generalisation to untreated items was demonstrated post-treatment in a participant with moderate mixed fluent aphasia and mostly phonological errors. Number of words and degree of informativeness in the picture description task also increased. Results remained at a 10-week follow-up. A participant with severe conduction aphasia and mainly semantic errors in the pre-training assessment also demonstrated a small increase in correct confrontation naming post-treatment. Some aspects of functional communication skills were rated slightly higher, but both participants perceived the negative impact of the aphasia to be greater post-treatment.

Conclusions: Phonological component analysis may be used in training of both objects and actions. However, aphasia severity and underlying deficits most likely influence the results and further research is warranted to explore the training effects.

Keywords: Aphasia, Anomia, Naming treatment, Generalisation

\section{INTRODUCTION}

Aphasia induced by neurological injury or disease such as stroke or traumatic brain injury prevents people from conveying wishes and needs and from participating in everyday communication. Anomia, or word retrieval difficulties, is a persistent symptom in essentially all types of aphasia [1]. The ability to name, for example, objects and actions varies between individuals and may be affected to different degrees depending on location of the brain damage [2-4] as well as by linguistic factors and the frequency of occurrence of the words to be produced [5].

The targets of anomia treatment may vary, but they often concern either the semantic content or the sound structure of the lexeme of the words [6]. Typically, nouns are

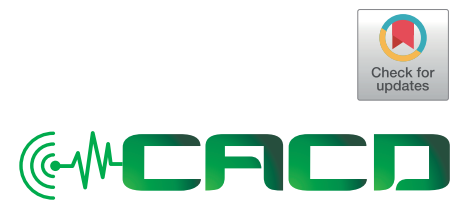

Received: June 29, 2018 Accepted: August 25, 2018

\section{Correspondence:}

Joana Kristensson

Institute of Neuroscience and Physiology, Division of Speech and Language Pathology, Sahlgrenska Academy, University of Gothenburg, Box 452, SE-405 30, Gothenburg, Sweden Tel: +4631786 6885

E-mail: joana.kristensson@gu.se

(C) 2018 The Korean Association of SpeechLanguage Pathologists

This is an Open Access article distributed under the terms of the Creative Commons Attribution NonCommercial License (http://creativecommons.org/ licenses/by-nc/4.0/) which permits unrestricted noncommercial use, distribution, and reproduction in any medium, provided the original work is properly cited. 
targeted in word-finding therapies. Effects of training of verbs have also been documented but have been reported to be smaller and generalisation effects harder to achieve compared with the effects of training of nouns [7].

Structured, multi-modal treatment methods comprising several components incorporate factors described as important for achieving treatment effects [8]. The optimal amount of training in relation to anomia has not yet been defined, but high-intensive treatment is generally considered more effective than low-intensive if tolerable to the participant [9].

Semantic feature analysis (SFA) [10] is an established and effective method for treating naming difficulties $[8,11,12]$. SFA has been used in several studies to treat persons with anomia after stroke [11,13-19] with positive effects on word retrieval for treated items and, to some extent, for untreated items.

SFA is based on the theory of spreading activation [20], according to which words are organised in semantic networks. When a concept is activated, the activation spreads to interlinked nodes that share the same features. As features of a specific word are activated, it reaches the threshold level for word production, making the correct word retrieval more likely to occur.

Corresponding to SFA, a phonological equivalent was designed in phonological components analysis (PCA) [21]. Effects of training using PCA have been explored with promising results. In one study, seven out of 10 participants (five with Broca's aphasia, one with anomia and one with mixed nonfluent aphasia) improved their ability to name treated items and they all maintained some level of treatment effects at follow-up four weeks later [21]. The three participants who did not improve their naming ability from treatment had severe naming impairments.

The emphasis on the importance of making active choices, i.e., actively providing appropriate responses, in the treatment of naming and word production, as present in SFA, is retained in PCA. Other components proposed to be important in effective treatment, such as self-generation of target, choice of target and cued responses [22], are all possible to incorporate in PCA. The participant is active during PCA treatment, generating or making active choices of phonological components of the target words. The active choice is argued to contribute to deeper processing of the task at hand and longer-lasting treatment effects on word retrieval [22]. Preliminary neuroimaging data further indicate that the activation of cognitive functions, as in making an active choice, is important for successful treatment of anomia. In a study with "choice" or "no choice" conditions, all five participants demonstrated treatment effects, but only the participant from the "choice" condition showed a change in neural activation [23].

A comparison between the SFA and PCA methods [16] showed that both methods are effective in improving naming abilities in individuals with aphasia. The results also indicated that PCA was beneficial for most participants regardless of whether the underlying deficit was phonological or semantic in nature. In the study, all eight participants received both SFA and PCA. Seven out of eight participants, all with mild or moderate aphasia of either anomic or conduction type, significantly improved their ability to name items treated with PCA. All but one maintained the results at follow-up. For items treated with SFA, only four of eight participants improved their naming, and three of them maintained the improvements at follow-up. Some of the participants demonstrated generalisation to connected speech as either their mean length of utterances (MLU) or the total number of utterances, or both, increased. In another study PCA treatment reduced the amount of neologisms for both treated and untreated items in a participant with jargon aphasia, apart from increasing naming ability for treated items [24].

Using fMRI, activation of various regions in the brain was identified as important for treatment outcome in PCA and SFA treatment [25]. For SFA treatment, pre-treatment activity in the left caudate, which is involved in monitoring semantic competition, was related to greater treatment outcome. For PCA treatment, the corresponding areas were the left supramarginal gyrus and the right precuneus. It was suggested that treatment targeting spared abilities is to be preferred to treatment targeting impaired processing.

Treatment effects on picture naming ability were demonstrated for treated items in two participants with Wernicke's and Broca's aphasia, respectively [26]. A modified version of PCA, using three of the original five features (rhyme, first sound and number of syllables) along with a modified SFA approach, was applied. The SFA approach seemed to be relatively more effective for the participant with Wernicke's aphasia, while the PCA approach initially seemed to be more effective for the participant with Broca's aphasia. However, the effect of SFA was longer lasting for both participants. A modest generalisation of effects to naming of untreated items was obtained for the participant with Broca's aphasia.

Another comparison of the effectiveness of SFA and PCA concluded that there is a direct link between underlying deficit and response to treatment [27]. PCA was more effective for 
Persian-speaking persons with underlying phonological deficits, while SFA was more effective for persons with semantic deficits.

Generalisation has proved difficult to achieve in many treatment studies. Examining the underlying deficit and relative strengths and weaknesses can possibly predict a person's likelihood of showing generalisation effects after anomia therapy [28]. Fifteen of 16 participants improved their ability to name treated items after eight weeks of phonological cuing therapy. Only the three participants with relatively good semantic processing and poorer phonological encoding demonstrated generalisation to untreated items.

All of the studies mentioned above used only objects as training items in the PCA. Hence, there is a need for studying the training effect of naming action words as well.

Apart from measuring the ability to name single words in a confrontation-naming task, additional outcome measurements should be considered to cover more aspects of importance to the participants [29]. Thus, a measurement of the effectiveness and content in connected speech is valuable and of great interest in the assessment of treatment effects after naming treatment. Communicating effectively in everyday life usually requires the ability to express oneself in connected speech in addition to one-word naming ability. There are established methods that have been used to analyse quality of connected speech, including content unit analysis [30] or counting correct information units (CIU) and frequency of words [31]. The CIU analysis method has been applied to assess results in various connected speech tasks such as giving personal or procedural information and in picture description tasks [13-17,32].

Although the effectiveness of PCA treatment for naming ability has been explored in a few single-subject studies, more research is called for to further explore the training effects. There is a lack of studies on the naming of action words besides objects and on the effect of connected speech. Hence, the aim of the current study is to evaluate the effects of PCA in two participants with aphasia with different degrees of severity and underlying deficits by attempting to replicate the results from the original study of PCA [21]. Additionally, the aim is to evaluate the effects of PCA on confrontation naming of both objects and action words. Furthermore, effects on connected speech and self-perceived functional communication will be explored.

\section{METHODS}

The present study uses a case study design with chronological time series analysis [33] and is approved by the Regional Ethical Board of Gothenburg, Sweden (ref.no. 506-16). The participants gave written consent after receiving oral and written information.

\section{Participants}

Two individuals, one woman and one man, participated in the study. Both were mono-lingual outpatients. At the time of the treatment, the woman ( $\mathrm{P} 1$ ) was 76 years old and the man (P2) 72. They had 11 and 20 years of education, and both were retired from employment in health care. Both participants were right-handed and met the inclusion criteria of being a native Swedish speaker, at least one year post-stroke and having no other known neurological injury or illness, no interfering presence of apraxia of speech, enough stamina to endure intensive treatment and sufficient vision, hearing and auditory comprehension to be able to perceive and comprehend instructions and participate in the treatment sessions.

Prior to the study, a general language assessment was performed by an experienced independent speech and language pathologist using the Swedish standardised aphasia test Neurolingvistisk Afasiundersökning [34,35]. The overall score, called the A-ning index, ranges between 0.0 and 5.0. A lower score indicates more severe aphasia. A-ning subtests target various aspects of language ability at single-word, sentence and text level. Each subtest is scored 0.0-5.0. Based on the assessment, both participants were diagnosed with anomia. P1 had moderate mixed fluent aphasia and P2 severe conduction aphasia (Table 1).

Prior to treatment, the nature of the underlying naming deficits was determined using the naming error analysis and subtests in a Swedish translation of the Psycholinguistic assessments of language processing in aphasia (PALPA) [36]. The performance of both participants was in the normal range, i.e., 90\% for Spoken Word-Picture Matching (subtest 47) and $100 \%$ for Written Word-Picture Matching (subtest 48) in PALPA. Both participants made twice as many errors on items with high imageability in subtest 49 (total score P1 80\% and P2 93\%), suggesting that the difficulties are modal-specific rather than an indication of a central semantic difficulty. The fact that both participants demonstrated quite good reading comprehension supports this assumption. The performance for both participants, $93 \%$ and $87 \%$ respectively, was 
Table 1. Background information and pre-treatment naming ability for the participants

\begin{tabular}{|c|c|c|}
\hline & Participant 1 & Participant 2 \\
\hline Age & 76 & 72 \\
\hline Sex & Female & Male \\
\hline Lesion & Large infarct left parietal-occipital & Large haemorrhage basal ganglia, oedema \\
\hline Years post-stroke & 2 & 5 \\
\hline Aphasia type & Mixed fluent & Conduction \\
\hline Aphasia severity & Moderate & Severe-moderate \\
\hline A-ningindex (max 5.0) & 3.5 & 2.8 \\
\hline Repetition & 2.1 & 3.3 \\
\hline Informative speech & 2.2 & 2.5 \\
\hline Auditory comprehension & 4.0 & 3.1 \\
\hline Reading comprehension & 4.5 & 4.3 \\
\hline Reading (aloud) & 4.5 & 3.0 \\
\hline Writing & 3.6 & 0.6 \\
\hline Object and Action Naming Battery & $43 \%$ & $0 \%$ \\
\hline Phonologic word fluency: Total (F, A, S) & $20(5,8,7)$ & $2(1,1,0)$ \\
\hline (Swedish norms for age, sex and education level. Total) & $(41.9 \pm 15.6)$ & $(42.3 \pm 10.6)$ \\
\hline Semantic word fluency: Animals & 5 & 1 \\
\hline (Swedish norms for age, sex and education level) & $(18.8 \pm 5.6)$ & $(20.9 \pm 7.1)$ \\
\hline Semantic word fluency: Verbs & 6 & 2 \\
\hline (Swedish norms for age, sex and education level) & $(17.9 \pm 5.9)$ & $(18.1 \pm 6.0)$ \\
\hline
\end{tabular}

below cut-off for normal controls in the Spoken Word-Written Word matching subtest (52), suggesting an underlying conceptual and/or lexical semantic impairment contributing to their word-finding difficulties. All errors in Semantic Word Association subtest (51) (total score P1 93\% and P2 80\%) were made on semantically related distractors.

To establish the pre-treatment naming ability and select treatment words the instrument An Object and Action Naming Battery (OANB) $[37,38]$ was used. The stimuli consisted of 160 black-and-white line drawings of objects (80) and actions (80). The selection of the picture material had been adapted to the Swedish language context and pre-tested on a group of non-brain-damaged native Swedes $(n=104)$ to ensure they were easily perceived, unambiguous representations of the targeted words $[39,40]$. The Swedish frequency of each word was determined [41] and the number of syllables and presence of consonant clusters were identified. Each participant was asked to name each of the 160 drawings in three consecutive individual sessions. No cuing or feedback was provided. For a response to be scored as correct, the participant had to produce it without any errors within 20 seconds.

Pre-treatment, P1's naming ability on OANB was $43 \%$. Her naming errors consisted of $16 \%$ semantic errors, $28 \%$ phonological errors, $1 \%$ omission and $12 \%$ circumlocutions; Table 1 . P2 did not manage to name any of the pictures in OANB correctly. His responses consisted of over 99\% non-verbal (gestures) responses combined with stereotypical or incomprehensible phrases and $0.5 \%$ semantic paraphasic errors.

The errors in the naming tasks were both semantic and phonological in nature for P1. For P2, they were semantic in nature but affected by a mild apraxia. In sum, the results suggest an underlying deficit in the connection of the lexical/phonological level for P1 and at the lexical semantic level for P2.

The phonological verbal fluency test FAS [42], in which the participant is asked to name words beginning with, in turn, the designated letters $\mathrm{F}, \mathrm{A}$, and $\mathrm{S}$ for one minute per letter was administered prior to intervention. In addition, semantic fluency was measured by a task where the participants were asked to name words belonging to the categories "animals" and "verbs" for one minute per category. The task was assessed according to the Swedish Norms [43] (Table 1).

\section{Intervention}

The treatment schedule was replicated from the one pre- 
sented by Leonard and colleagues (2008) [21], yet with some differences. A baseline was established in three consecutive sessions for each participant before the treatment started with the set 1 pictures. The order of presentation of the target words was randomised within each set every time. Both participants in the present study had anomia regardless of word class and both objects and action words were used, unlike in Leonard et al. [21], where only objects were used. In the present study, individual treatment sessions delivered by a speech-language pathologist (the first author) at an outpatient clinic in Sweden, occurred five times a week in contrast to three times a week in Leonard et al. [21].

The participants were asked to choose words they would like to use in the treatment from words they were unable to name correctly in at least two of the three sessions. All treatment words chosen by the participants were in fact words they were unable to name in all three sessions. Forty of the chosen words were divided into four sets (10 words in each). Each set consisted of five pictures of actions and five of objects, except for P1's Set 1, which consisted of four pictures of actions and six of objects. For each participant, the sets were matched to be as equivalent as possible in terms of frequency, consonant cluster, number of syllables and number of phonemes. An extra set of words freely chosen by each participant was added purely for motivational reasons. P1 chose for instance names of the months and numerical values. P2's personal set consisted almost entirely of names of loved ones. The participants' extra sets were not included as measures of naming ability.

The PCA protocol used by Leonard and colleagues (2008) [21] was followed in the treatment sessions. Thus, a table white board with a square for each of five different phonological components was placed in front of the participant. The participant was asked to name a picture representing a target word placed in the centre of the table white board by answering the question, "What is this" or "What is he/she doing?". Regardless of whether the participants were able to name the target word correctly, they were asked to identify each of the following components in a set order:

1. Rhyme (What does this rhyme with?)

2. First sound (What sound does it start with?)

3. First sound associate (What other word starts with the same sound?)

4. Final sound (What sound does it end with?)

5. Number of syllables (How many beats does the word have?)
Parenthetically, verbs in the present tense in Swedish end in -r, -er, -ar, -or or -s. If the participant was unable to name a component, a list of up to three suggestions was provided orally and in writing by the clinician for the participant to choose from. When a correct component was provided, the clinician wrote it on the chart in the corresponding square. When all squares had been filled, the participant was asked to name the target word again. A correct response was acknowledged by the clinician saying "Yes, that's right. It's a..." or "Yes, that's right. She/He ...." If an answer was incorrect, the clinician provided the right answer and asked the participant to repeat it. Irrespective of whether the participant was able to name a picture, all phonological components were reviewed by the clinician and the participant was once again asked to name the picture. The responses were handled in the same manner as described earlier. In this way, each target word was provided orally twice in every session regardless of whether the participant was able to name it independently or with support. The target word was never presented in written form.

At each session, in total 17 for P1 and 20 for P2, it was ensured that all the training words in the set presently used were trained. If there was any time left, words from the self-chosen extra set were added.

\section{Outcome measures and procedures}

Treatment sessions were approximately one hour in duration and occurred five times a week. Probes of the ability to name treated items were conducted at the end of every second or third session, in accordance with the design used in Leonard et al. [21]. The items within each set were presented in randomised order. Strategies from the PCA scheme were not used and no cuing was provided. Since the aim of this training was to improve accurate and effective naming of specific words, chosen by the participants, no synonyms or alternative responses were accepted as correct responses. Likewise, no phonological errors were accepted and the time limit was set to 10 seconds per item in the probe sessions. Hence, responses were scored as correct when the participant gave a semantically and phonologically correct or self-corrected, answer within 10 seconds. Scoring was made on-line by the first author in order to keep track of success rate, which determined when to start the training of each set. When $80 \%$ of the words of the treated set were correctly named in two consecutive probe sessions, or after a maximum of 15 treatment sessions, the set was set aside and treatment on a new set initiated. The treatment was discontinued if a participant did not 
achieve $40 \%$ accuracy on set 2 .

Probes of the ability to name treated items were made repeatedly during baseline and intervention, directly after treatment and at follow-up ten weeks after treatment. The sets of untreated items were probed for accuracy at the end of every third treatment session to minimise the effect of repeated measure. Correspondingly, the discontinued sets were probed for naming accuracy in every third session in order to examine maintenance effects. All probes used for the analysis were later scored from video-recordings by a rater blinded for when the recordings were made.

To measure generalisation to connected speech, the participants were asked to describe the Cookie Theft picture from The Boston Diagnostic Aphasia Examination (BDAE) [44]. The PCA chart was not used during this task and no prompting was given. The Cookie Theft task was administered before, directly after treatment and at follow-up 10 weeks later. The participants' responses, captured on video, were analysed by two speech and language therapists other than the original examiner and blinded to when the recordings were made. The analyses included number of words and words per minute as well as number and percentage of correct information units (\%CIU). Two calculations were made, i.e., for the entire description and for the first minute of the description, to examine any difference in the ability to quickly and effectively produce correct information. The assessment of connected speech was based on published guidelines [31]. The method was used to distinguish accurate, intelligible, relevant and informative words in connected speech from irrelevant and uninformative verbal production.

To acquire the all-important first-hand information about how the person with aphasia perceives his or her own communicative abilities and to explore if a relatively short intervention is reflected in the ratings of life quality and self-perceived communication the participants were asked to complete a questionnaire. The Communication Outcomes after Stroke (COAST) scale [45] is a valid and reliable questionnaire that includes picture support to enable persons with aphasia to rate their self-perceived functional communication skills and quality of life on a 5-point Likert response scale. Twenty items, e.g., "In the past week or so, how well could you have a short conversation with an unfamiliar person?" and "How often does confidence about communicating affect what you do?" are scored using a 0 (worst scenario) to 4 (best scenario) scale. Corresponding information was obtained from the participants' significant others, using Carer-COAST [46]. The
COAST and Carer-COAST questionnaires were administered before, directly after treatment and at follow-up 10 weeks later.

\section{Data analysis}

Percentage of non-overlapping data (PND) [47] was used in the analysis of treatment effects on naming ability. That is, the percentage of treatment data points exceeding the highest baseline point was calculated and established guidelines for interpretation of degree of effect were used: PND $90 \%$ or greater $=$ very effective; $70 \%$ to $89 \%=$ effective; less than $70 \%=$ questionable or ineffective [48].

Effect size were calculated for the dependent variables using $d$-index [49]. The calculations were based on all results from the baseline phase and the last three measurements in the intervention phase. The relative strength of the treatment effect was interpreted as a small, medium or large effect size, corresponding to a $d$-index of $2.6,3.9$, and 5.8 , respectively $[49,50]$.

For the analysis of connected speech in the Cookie Theft task, CIUs were calculated and compared to detect possible differences in results before vs. after intervention. The entire verbal description and the first minute of the description were analysed.

All probes were video recorded, coded and randomised. The recordings were scored by a speech and language therapist other than the original examiner and blinded to when the recording was made. To analyse reliability, using point-topoint percent agreement, $30 \%$ of the material was randomly selected to be scored twice and by two different raters. The intra-rater reliability for naming was nearly perfect with $99 \%$ agreement. To obtain a measure of inter-rater reliability, the blinded assessor's scores were compared with the researcher's/first author's assessments. This value also amounted to 99\%. The inter-rater reliability for word count between the two assessors was $99 \%$ and for CIU count $92 \%$. The intra-rater reliability was $100 \%$ for word count and $95 \%$ for CIU.

\section{RESULTS}

The ability to name treated items increased during intervention for P1, i.e., the woman with moderate mixed fluent aphasia. Although there was a slightly unstable baseline, there was an increase in naming ability after the intervention onset for two of three treated sets, while the fourth, untreated, set remained at a low level of successful naming; Figure 1. During the training of set 2, Pl's ability to name the untreated items in 


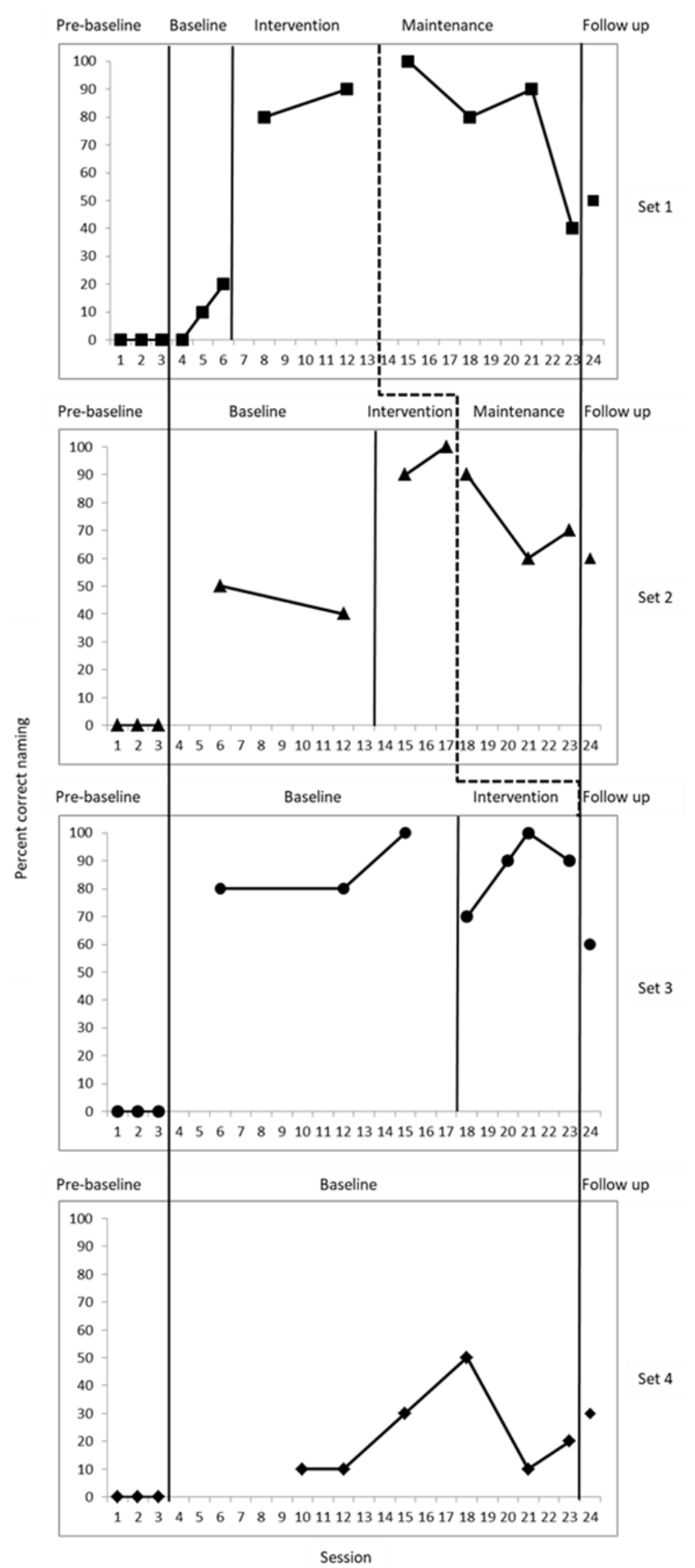

Figure 1. Naming ability in treated (set 1-3) and untreated items (set 4) for Participant 1.

Set 3 also increased to the $80 \%$ level while still in the baseline phase, and the fourth set of untreated items was introduced. All treated sets reached $100 \%$ accuracy while the untreated set did not. Although the results tended to decrease when the training of each set is withdrawn, they remained above the baseline results at the follow-up session several weeks after the training ended.

When calculating effect sizes as $d$-index, the treatment effect for P1 was large for set $1(d=6.8)$ and set $2(d=4)$ but $(d=0.1)$ for set 3 .

The percentage of non-overlapping data (PND) for P1 was $100 \%$ for sets 1 and 2 . According to the guidelines for interpretation of PND as a measure of treatment effects [48], the treatment provided in the present study would be interpreted as very effective for $\mathrm{P} 1$ for 2 of 3 sets. Bearing in mind the naming ability in the pre-baseline phase, which was 0 for all sets, the ability to name treated items increased considerably.

For P1, the CIU analysis from the description of the Cookie Theft picture indicated an increase in informative statements, fewer empty words and a more effective way of conveying information. The total number of CIUs increased by $42 \%$ after intervention (from 92 to 130), and the total number of words increased by $29 \%$ (from 151 to 195), Table 2 . When limiting the analyses to the first minute of the description, P1 was able to convey 128 words and 78 CIUs after intervention compared with 103 words and 56 CIUs before, corresponding to increases of $25 \%$ and $39 \%$, respectively. The improvements in total number of words and words per minute, but not in CIUs, were maintained at the follow-up 10 weeks after treatment.

The overall COAST ratings stayed relatively unchanged throughout all probe sessions. At the 10-week follow-up session, $\mathrm{P} 1$ reported an increased ability to communicate with an unknown person, to follow a change of topic, to use longer sentences as well as other means of communication, to read and write and to handle money. At the same time, she reported decreased communicative confidence, increased concern and greater negative impact on interests/hobbies as well as a slight overall decrease in quality of life. While P1 total ratings slightly increased at follow-up, her next-of-kin's ratings in Carer-COAST slightly decreased, resulting in a shared estimation of functional communication at that time.

The naming ability did not differ significantly with respect to word class. The naming of actions and objects was interrelated for both participants during the sessions; Figure 3.

For P2, the man with severe conduction aphasia, the ability to name treated items increased from $10 \%$ to $20 \%$ in Set 1 and from $0 \%$ to $20 \%$ in Set 2 . This result was stable throughout the maintenance stage for both sets and at follow-up for Set 1 (Figure 2).

For P2, the PND was $71 \%$ for Set 1 and $100 \%$ for Set 2 . Ac- 
Table 2. Picture description task for participant 1 (P1) and participant 2 (P2)

\begin{tabular}{|c|c|c|c|c|c|c|}
\hline \multirow{2}{*}{$\begin{array}{l}\text { Picture description } \\
\text { Total and (during first minute) }\end{array}$} & \multicolumn{2}{|c|}{ Before } & \multicolumn{2}{|c|}{ After } & \multicolumn{2}{|c|}{ Follow up } \\
\hline & P1 & $\mathrm{P} 2$ & $\mathrm{P} 1$ & $\mathrm{P} 2$ & P1 & $\mathrm{P} 2$ \\
\hline Number of words & $151(103)$ & $345(83)$ & 195 (128) & - & $189(114)$ & 268 (99) \\
\hline Number of CIUs & $92(56)$ & $40(11)$ & $130(78)$ & - & $95(69)$ & $12(7)$ \\
\hline Words per minute & 109 (103) & $87(83)$ & $111(128)$ & - & $137(114)$ & 113 (99) \\
\hline Percent CIU & $61(54)$ & $12(13)$ & $67(61)$ & - & $50(61)$ & $5(7)$ \\
\hline CIUs per minute & $67(56)$ & $10(11)$ & $74(78)$ & - & $69(69)$ & $13(7)$ \\
\hline
\end{tabular}

- , Data missing; CIU, correct information unit.

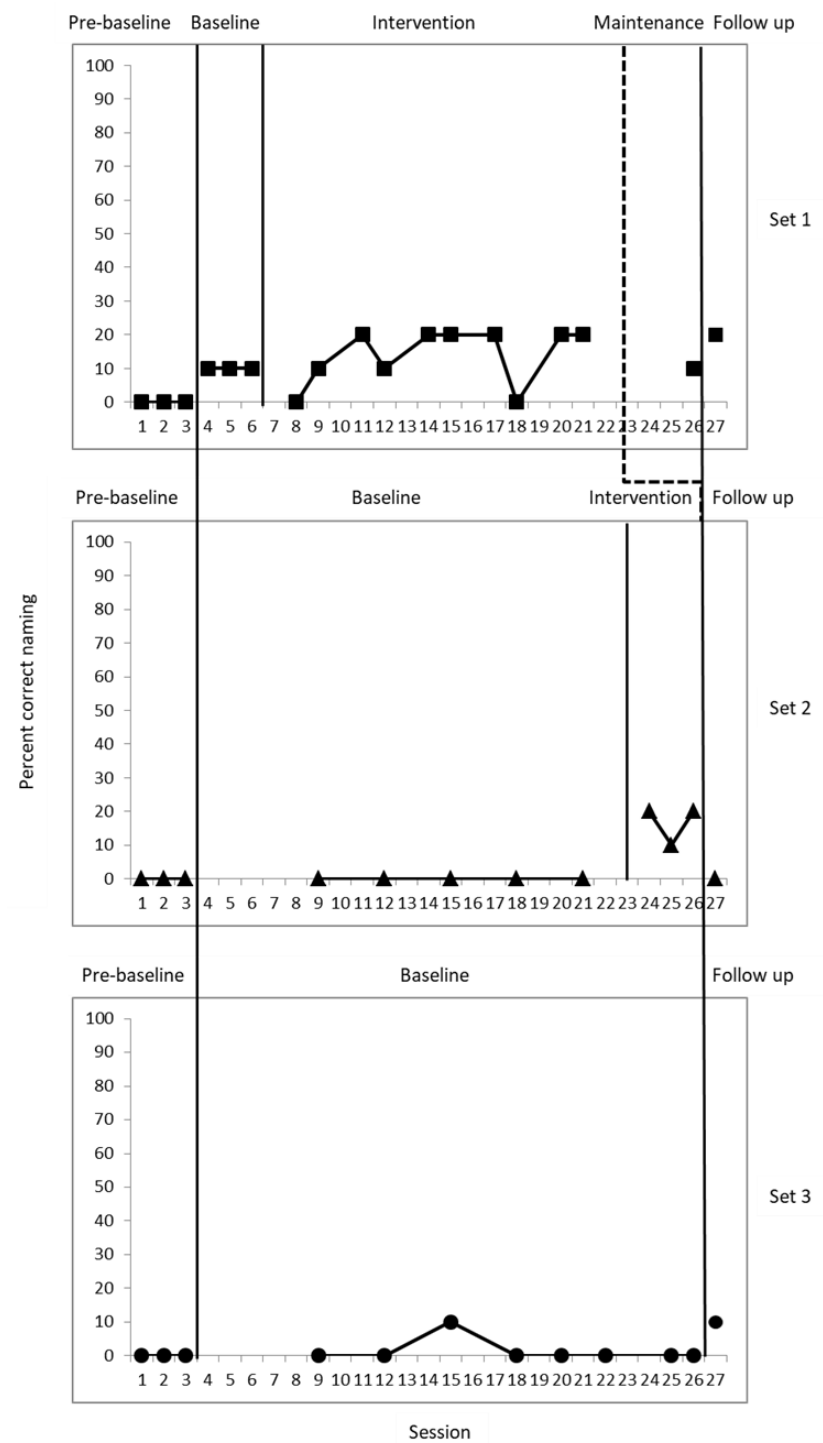

Figure 2. Naming ability in treated (set 1-2) and untreated items (set 3) for Participant 2.

cording to the guidelines for interpretation of PND as a measure of treatment effects [48], the treatment provided in the present study would be interpreted as effective to very effec-
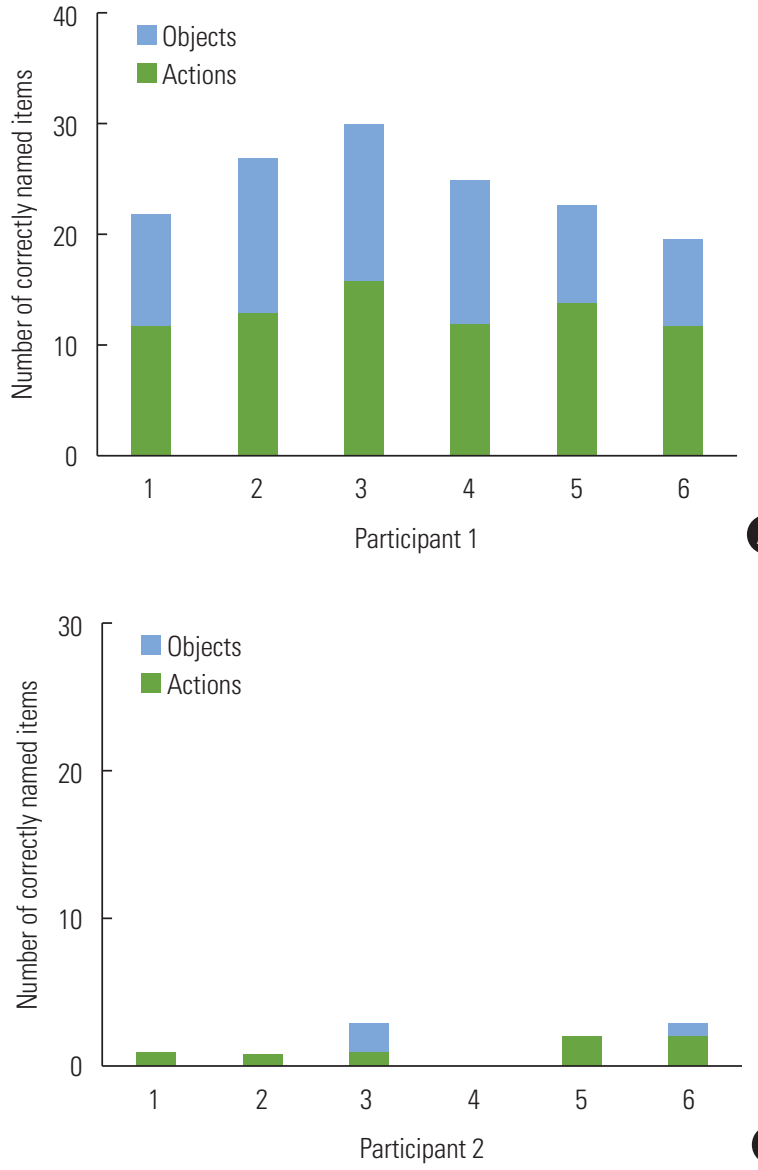

Figure 3. Correct naming of action and objects on sessions when all sets were probed.

tive for this participant. The calculation of effect size was not possible for $\mathrm{P} 2$ due to stable baselines and absence of maintenance phase.

Unfortunately, due to unforeseen circumstances that prevented P2 to participate in further probe sessions at that time, data is missing from immediately after intervention. No improvement of informativeness in connected speech could be seen at follow-up. On the contrary, at follow-up, a $29 \%$ de- 

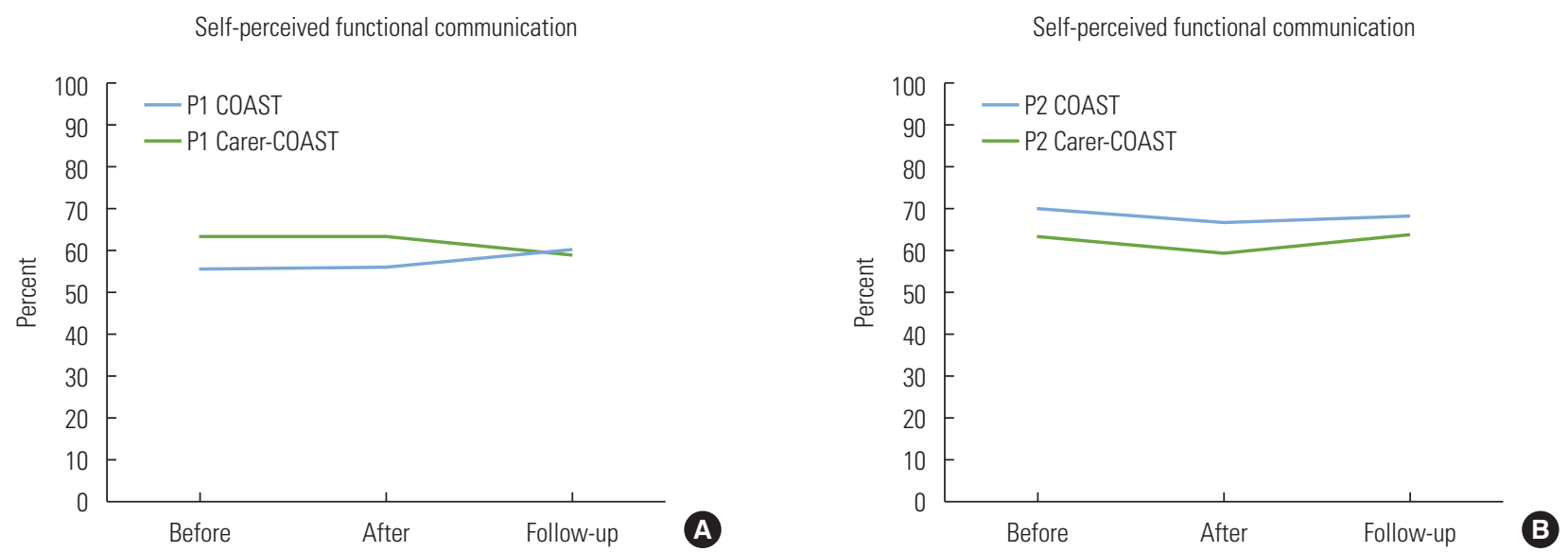

Figure 4. Self-perceived functional communication rated by the participants (COAST) and their next-of-kins (Carer-COAST).

crease in total number of words was recorded. The total number of CIUs decreased by one third and \%CIU halved, Table 2. The numbers of CUIs per minute were small and relatively constant. However, in the first minute, there was a $19 \%$ increase in total number of words and words per minute.

P2's overall COAST ratings and those of his next-of-kin mirrored each other throughout, showing a minor decline immediately after intervention only to return to the initial level at follow-up (Figure 4). P2 reported an increased ability to use longer sentences at follow-up, but he also reported a more negative overall view of his communication skills as well as a greater negative impact on family and social life.

As a curiosity, both $\mathrm{P} 1$ and $\mathrm{P} 2$ reached $100 \%$ correct naming on the set of highly personalised words at least once when probed during treatment sessions. As there is no baseline data on the personalised words, they are not included in the measuring of outcome but will be discussed separately below. However, both participants stated prior to the intervention that the words chosen were difficult to name. In the beginning of the intervention, they were unable to name the items in the personalised set. However, P1 named 50\%, 90\%, and $100 \%$ correctly in Session 5, at the end of the intervention and at follow-up, respectively. P2 named one item correctly in the second session and 0 in the third. In Session 6, he named 50\% correctly, and in session $16100 \%$. Interestingly, this was the only set in which he scored $100 \%$. However, due to the lack of baseline, this needs to be considered with caution.

\section{DISCUSSION AND CONCLUSIONS}

PCA [21] was used in treatment of both objects and actions in this single subject study with two participants. The aim was to evaluate the use of PCA in improving one-word confrontation naming ability, connected speech and perceived functional communication skills.

Measures obtained after the start of the treatment showed an increase in correct production of treated words in one of the two participants. Compared with the untreated set, all treated sets improved for $\mathrm{P} 1$. In set 1 , the naming accuracy was twice as high compared with the untreated set 4 at the follow-up session 10 weeks post-intervention. During treatment sessions, the naming ability of sets 2 and 3 was improving faster than that of set1, which is equivalent to a finding by Leonard and colleagues (2008) [21]. Furthermore, sets 2 and 3 were maintained better than set 1 at follow-up. Though there was an immediate decline in results at training withdrawal, the results returned to a level higher than baseline at the follow-up session several weeks post-training for sets 1-3. This might imply a synergistic effect, which enhances generalisation. That is, the method may promote learned use of a strategy, which may help boost the training effect in subsequent sets. However, it seems that this effect alone is not sufficient but that there is a need to also focus on specific words in order to improve the naming of them.

As in the study by Leonard and colleagues (2008) [21] the person with severe aphasia (P2) shows only a minor increase in production of correct words in the present study. His small improvement on set 1 was achieved after a greater number of training sessions than P1 used for a set, and the result was not maintained at follow-up. This finding suggests that persons with severe aphasia might benefit from PCA but need a greater amount of training over a sustained period. Even for $\mathrm{P} 1$, there is an instant decline in results when the training stopped. To increase the quantity and length of training in an 
effort to maintain acquired abilities, possibly a prolonged maintenance phase with sustained massed practice and/or added self-practice may be good. A greater number of therapy sessions over a prolonged period has been shown to yield better and longer lasting therapy effects [9,51]. Since the PCA method is straightforward and fairly easy to perform, the training may very well be continued after the initial intervention period, for instance administered by a volunteer after receiving tutoring and pre-made training material.

The importance of personalised items is raised in several studies [52-54]. Personally relevant items were acquired more successfully and naming ability was significantly better for these than for other words and phrases. In order to increase the influence and participation of the participants in the present study, all training words were chosen by the participants. The training sets were selected from a predetermined set of words to enable matching, comparison and measuring outcome. One additional set was composed by words freely chosen by each participant and not matched in any way. This set was included in the training material mostly for motivational reasons, since the other items were inherently difficult to name and would inevitably lead to struggle and failure during training sessions. This was true particularly for P2, who had severe conduction aphasia. He chose the names of his family members for his personal set, and this was indeed an appreciated element in the training. However, it should be noted that P2 was highly motivated throughout the treatment, regardless of training item. Still, there was a difference in speed and quantity in P2's ability to name the set of freely chosen words compared with the other sets. Perhaps higher emotional dedication was the key to reaching results. However, one cannot rule out the possibility that the results indicate mere fluctuation. Though only speculative as the motivational words lack baseline measures, they may still provide interesting insight regarding the importance of choice of material. Maybe people, and people with severe aphasia in particular, should not only choose their own treatment material but also be encouraged to choose material with close personal meaning. A higher degree of motivation, engagement, deep-level processing and active participation will more likely lead to better treatment effects.

For P1, who had moderate and fluent aphasia, there was a small difference in average naming ability between the sets, i.e., $84 \%$ for the freely chosen words and on average $57 \%$ for the others. She chose the names of the months and numbers for her personalised set. The PCA method was difficult to ap- ply to these words. For example, the feature rhyme seemed to be confusing rather than helpful, since, for example, a natural rhyme for December is November. The number 465 is phonologically not very distinctive from 455 , in terms of the features "first sound, last sound, same first sound, rhyme and number of syllables". From the observations made during this study, it can be recommended that participants should be encouraged to choose their own training words, and to do so in abundance, giving the therapist room to exclude words unsuited for the method. Still, the PCA has an advantage over SFA in that virtually any word can be used, regardless of word class. Although this study concerns nouns and verbs both participants chose words from other word classes as well, including numerals and proper names. Based on clinical experience, being able to say the names of loved ones is a common wish in patients.

Generalisation of the training effect from specific words to naming ability in general is highly desirable in order to be useful in everyday communication. One strength of SFA [10] is that the method can be used as a strategy in case of anomia in everyday conversations. Neither participant in the present PCA study spontaneously used the elements in PCA as a strategy in occurrences of anomia. Self-prompting in PCA can consist of tapping the rhythm of a word or sounding the initial of the target word [16]. Providing phonological cues has been found to effectively facilitate naming performance [55] but to be able to cue depend on the ability to grasp the phonological aspects of the sought-after word. This may make this strategy less useful than the semantically related strategy provided in SFA, which enables a description of the general meaning rather than the form of a specific word.

For P1, two measurements of connected speech increased after intervention compared with before: number of words and number of CIUs. When comparing the production during the first minute of the performance of the task before and after intervention, an improvement in effectiveness can be noted. Thus, it seems that P1 was able to give the same amount of correct information in less time than before intervention, and this effect was maintained at the 10-week follow-up.

There is no data on P2's post-intervention ability in the picture description task. However, since P2 did not improve much in the one-word picture naming, no improvement in connected speech would be expected. At the 10-week followup, there was actually a decrease in total number of words. This could be a desirable result in situations of excessively fluent speech if accompanied by an increase of CIUs, but this 
was not the case here. CUIs per minute and \%CIU were relatively constant, which could indicate that $\mathrm{P} 2$ delivered the equivalent amount of relevant information in the same amount of time both before intervention and at follow-up but refrained from giving more incidental information at followup. However, the small numbers make it difficult to draw any assertive conclusions from this data.

The difference in change in production of correct words between the two participants in the measures obtained before and after treatment may be due to several factors. There is an obvious difference in aphasia severity. The participant with more severe aphasia did not improve as much as the participant with moderate aphasia did. The variation in treatment effect in individuals with different degrees of aphasia is in accordance with results from a study by Leonard and colleagues (2008). However, the participants also differed in underlying deficits. According to the results on PALPA, P2, i.e., the participant with predominately lexical-semantic deficits, did not improve to the same extent as $\mathrm{Pl}$, i.e., the participant with an underlying deficit in the connection of the lexical/phonological level. This may imply that P1 benefitted more from the treatment because it targeted the phonologic components, consistent with results in a previous study [27].

No obvious change was evident in the more complex measure of perceived functional communication. The overall COAST ratings stayed relatively unchanged throughout all probe sessions before intervention, directly after intervention and at the 10-week follow-up. However, although there is no effect on the overall score, there is a shift in P1's ratings after intervention. Communications skills such as the ability to follow changes in topic, to use longer sentences and other multimodal communication as well as to communicate better with unknown people were rated higher while communicative confidence and quality of life were rated lower. Similarly, P2 rated his own communication skills lower and experienced a greater negative impact on social and family life, but rated an increased ability to use longer sentences. However, using complex measures distal from what has been trained when assessing the outcomes of treatment studies can be complicated. Increased naming ability does not necessarily increase perceived communication skills and participation, but even if it does, it is difficult to reveal as several other factors in everyday life may have impact on self- reported outcome measures [56]. Although adapted to facilitate for persons with aphasia, the self-report instrument used in this study, COAST, might not be appropriate for capturing any or all possible changes in perceived communication skills in the participants. Furthermore, participating in a treatment program may bring insights about degree of functional communication skills. Thus, even if more proximal outcome measures indicate improvements with respect to the treated aspects, the treatment may also bring the remaining difficulties to the fore $[57,58]$. Correspondingly, this could be the reason for the minor decline in the ratings reported by the participants' next of kin in CarerCOAST [46].

Aphasia treatment can be effective in chronic aphasia, but the intervention must be given for a sufficient period of time $[9,59]$. The more or less stable results in COAST in general could reflect the fact that both participants have had aphasia for many years and that their everyday functional communication therefore was less likely to change after a relatively short period of intervention.

An interesting detail was the similarity between the results for the participants. P1's overall ratings were actually a little lower than P2's (Figure 4). Degree of aphasia does not seem to reflect the self-perceived difficulties in functional communication in this case.

Some limitations of the current study should be noted. First, this is a Phase 1 single subject study still exploring requirements of dosage and for whom the method is suitable [60]. There are only two participants and although the results provide valuable information about possible effects of PCA, the findings in the present and previous studies need to be replicated with a larger sample size in controlled trials to be able to generalise any results to a general population. Secondly, the lack of baseline for the extra set of training items hinders further exploration of possible differences in the ability to name items with varying personal relevance. Thirdly, the instability of the baseline is undoubtedly problematic and makes data interpretation difficult. Initial scoring was made on-line by the first author and intervention was initiated when the baseline was relatively stable. A slight difference in the scores by the blinded rater revealed a higher degree of instability in the baseline, which would have advocated a pro-longed baseline. Furthermore, in this study the aim was to replicate the case study design, which Leonard and colleagues (2008) [21] reports as a single subject design across behaviours. However, appropriate base line data (before the initiation of treatment with set 1 ) is missing for training sets $2-4$. Thus, the case study design used does not provide the high degree of control as a proper single subject design across behaviours would. This means that the implication of an increase in number of cor- 
rect words produced after treatment in this study must be interpreted with caution.

As the probing schedule in Leonard et al. [21] was followed, probes were taken at the end of sessions, not on separate occasions or at the beginning of sessions. In this way, probing of trained items was regarded as part of the training session and clinically relevant as a summary of the session. However, it would be preferable to probe at the beginning of sessions instead of in the end in order to be able to distinguish retention of acquired items from immediate training effect as well as to avoid effect of fatigue in the participants.

Additional information, such as the participants' perceptions of various qualitative aspects on consequences in everyday communication, would have been valuable. Additionally, the importance of careful selection of training material needs to be further explored.

In conclusion, this study indicates that PCA may be used in training of both objects and actions. Furthermore, the study indicates that treatment with PCA may result in an increase in number of correct words in confrontation naming of treated items. Additionally, it indicates that increases in the production of correct information units in connected speech can be achieved in individuals with moderate mixed fluent aphasia. However, aphasia severity and underlying deficits most likely influence the training effect. When selecting training material, it must be ensured that the items are suitable for both the participant and the training method. Further research is needed to examine what factors have the most influence in training effects.

\section{CONFLICT OF INTEREST}

The authors declare that they have no conflict of interests.

\section{REFERENCES}

1. Goodglass H, Wingfield A, editors. Anomia: neuroanatomical and cognitive correlates. Toronto: Academic Press; 1997.

2. Conroy P, Sage K, Lambon Ralph MA. Towards theory-driven therapies for aphasic verb impairments: A review of current theory and practice. Aphasiology, 2006;12:1159-1185.

3. Damasio AR, Tranel D. Nouns and verbs are retrieved with differently distributed neural systems. Neurobiology Proc Natl Acad Sci. USA. 1993;4957-4960.

4. Pulvermüller F, Lutzenberger W, Preissl H. Nouns and Verbs in the Intact Brain: evidence from Event-related Potentials and Highfrequency Cortical Responses. Cerebral Cortex. 1999;9:497-506.

5. Bastiaanse R, Wieling M, Wolthuis $\mathrm{N}$. The role of frequency in the retrieval of nouns and verbs in aphasia. Aphasiology. 2016;30: 1221-1239.

6. Wisenburn B, Mahoney K. A meta-analysis of word-finding treatments for aphasia. Aphasiology. 2009;23;11:1338-1352.

7. Webster J, Whitworth A. Treating verbs in aphasia: exploring the impact of therapy at the single word and sentence levels. International Journal of Language \& Communication Disorders. 2012;47: 619-636.

8. Nickels L. Therapy for naming disorders: revisiting, revising and reviewing. Aphasiology. 2002;16:935-979.

9. Brady MC, Kelly H, Godwin J, Enderby P, Campbell P. Speech and language therapy for aphasia following stroke. Cochrane Database of Systematic Reviews 2016(6). Art. No.: CD000425.

10. Massaro, M, Tompkins C. Feature analysis for treatment of communication disorders in traumatically brain-injured patients: an efficacy study. Clinical Aphasiology. 1994;22:245-256.

11. Boyle M. Semantic feature analysis treatment for aphasic word retrieval impairments: what's in a name? Top Stroke Rehabilitation. 2010;17:411-422.

12. Maddy KM, Capilouto GJ, McComas KL. The effectiveness of semantic feature analysis: an evidence-based systematic review. Annals Physical and Rehabilitation Medicine. 2014;57:254-267.

13. Boyle M. Semantic feature analysis treatment for anomia in two fluent aphasia syndromes. American Journal of Speech-Language Pathology. 2004;13:236-249.

14. Boyle M, Coelho CA. Application of a semantic feature analysis as a treatment for aphasic dysnomia. American Journal of SpeechLanguage Pathology. 1995;13:236-249.

15. Coelho CA, McHugh RE, Boyle M. Semantic feature analysis as a treatment for aphasic dysnomia: a replication. Aphasiology. 2000; 14:133-142.

16. van Hees S, Angwin A, McMahon K, Copland D. A comparison of semantic feature analysis and phonological components analysis for the treatment of naming impairments in aphasia. Neuropsychological Rehabilitation. 2013;23:102-132.

17. Wambaugh JL, Ferguson M. Application of semantic feature analysis to retrieval of action names in aphasia. Journal of Rehabilitation Research and Development. 2007;44:381-394.

18. Rider JD, Wright HH, Marshall RC, Page JL. Using semantic feature analysis to improve contextual discourse in adults with aphasia. American Journal of Speech-Language Pathology. 2008;17: 161-172.

19. Kristensson J, Behrns I, Saldert C. Effects on communication from intensive treatment with semantic feature analysis in aphasia. Aphasiology. 2015;29:466-487.

20. Collins AM, Loftus EF. A spreading-activation theory of semantic processing. Psychological Review. 1975;82:407-428.

21. Leonard C, Rochon E, Laird L. Treating naming impairments in aphasia: findings from a phonological components analysis treatment. Aphasiology. 2008;22:923-947.

22. Hickin J, Best W, Herbert R, Howard D, Osborne F. Phonological therapy for word-finding difficulties: a re-evaluation. Aphasiology. 2002;16:981-1000. 
Kristensson J, et al. Naming of objects and actions after PCA in aphasia

23. Leonard C, Laird L, Burianová H, Graham S, Grady C, Simic T, et al. Behavioural and neural changes after a "choice" therapy for naming deficits in aphasia: preliminary findings. Aphasiology. 2015;29:506-525.

24. Bose A. Phonological therapy in jargon aphasia: effects on naming and neologisms. International Journal of Language \& Communication Disorders. 2013;48:582-595.

25. van Hees S, McMahon K, Angwin A, de Zubicaray G, Copland DA. Neural activity associated with semantic versus phonological anomia treatments in aphasia. Brain and Language. 2014;129,4757.

26. Hashimoto N. The use of semantic- and phonological-based feature approaches to treat naming deficits in aphasia. Clinical Linguistics \& Phonetics. 2012;26:518-553.

27. Sadeghi Z, Baharloei N, Moddarres Zadeh A, Ghasisin L. Comparative effectiveness of semantic feature analysis (SFA) and phonological components analysis (PCA) for anomia treatment in persian speaking patients with aphasia. Iranian Rehabilitation Journal. 2017;15:259-268.

28. Best W, Greenwood A, Grassly J, Herbert R, Hickin J, Howard D. Aphasia rehabilitation: does generalisation from anomia therapy occur and is it predictable? A case series study. Cortex. 2013;49: 2345-2357.

29. Simmons-Mackie N, Threats TT, Kagan A. Outcome assessment in aphasia: a survey. Journal of Communication Disorders, 2005; 38:1-27.

30. Yorkston K, Beukelman D. An analysis of connected speech samples of aphasic and normal speakers. Journal of Speech and Hearing Disorders. 1980;45:27-36.

31. Nicholas LE, Brookshire RH. A system for quantifying the informativeness and efficiency of the connected speech of adults with aphasia. Journal of Speech, Language, and Hearing Research. April 1993;36:338-350.

32. Peach RK, Reuter KA. A discourse-based approach to semantic feature analysis for the treatment of aphasic word retrieval failures. Aphasiology. 2010;24:971-990.

33. Yin R. Case study research: design and methods (5th ed.). London: SAGE; 2014.

34. Lindström E, Werner C. A-ning - Neurolingvistisk Afasiundersökning. Stockholm: Ersta högskola - Ersta utbildningsinstitut; 1995 Swedish.

35. Lindström E, Werner C. A-ning - Neurolingvistisk Afasiundersökning. Standardisering. Stockholm: Ersta högskola - Ersta utbildningsinstitut; 2000 Swedish.

36. Kay J, Lesser R, Coltheart M. Psycholinguistic assessments of language processing in aphasia (PALPA). Hove, UK: Lawrence Erlbaum Associates Ltd.; 1992.

37. Masterson J, Druks J. Description of a set of 164 nouns and 102 verbs matched for printed word frequency, familiarity and age of acquisition. J. Neurolinguist. 1998;11:331-354.

38. Druks J, Masterson J. An object and action naming battery. Philadelphia: Psychology Press; 2000.

39. Andersson L, Larsfelt S. [Internet] Semantisk särdragsanalys: Be- handlingsmetodens effekt på benämningsförmågan hos tre personer med ordfinnandesvårigheter till följd av afasi. 2013. Sahlgrenska akademin. Göteborgs universitet.

40. Hellberg M, Kroon M. [Internet] Konfrontationsbenämning av substantiv och verb hos svensktalande vuxna utan anomi. Göteborg: Institutionen för neurovetenskap och fysiologi, Göteborgs universitet. 2014.

41. Corpora at Department of Linguistics, Gothenburg University. [Internet] 2002.

42. Lezak MD. Neuropsychological Assessment. Third edition. New York: Oxford University Press; 1995.

43. Tallberg IM, Ivachova E, Jones Tinghag K, Östberg P. Swedish norms for word fluency tests: fas, animals and verbs. Scandinavian Journal of Psychology. 2008;49:479-485.

44. Goodglass H, Kapland E. The Boston Diagnostic Aphasia Examination. (2nd ed.). Philadelphia, PA: Lea \& Febiger; 1983.

45. Long A.F, Hesketh A, Paszek G, Booth M, Bowen A. Development of a reliable, self-report outcome measure for pragmatic trials of communication therapy following stroke: the Communication Outcome after Stroke (COAST) scale. Clinical Rehabilitation. 2008;22:1083-1094.

46. Long A, Hesketh A, Bowen A. Communication outcome after stroke: a new measure of the carer's perspective. Clinical Rehabilitation. 2009;23:846-856.

47. Scruggs TE, Mastropieri MA, Casto G. The quantitative synthesis of single-subject research: methodology and validation. Remedial and Special Education. 1987;8:24-33.

48. Scruggs TE, Mastropieri MA. PND at 25: past, present, and future trends in summarizing single-subject research. Remedial and Special Education. 2012;34:9-19.

49. Beeson P, Robey R. Evaluating single-subject treatment research: lessons learned from the aphasia literature. Neuropsychology Review. 2006;16:161-169.

50. Beeson PM, Egnor H. Combining treatment for written and spoken naming. Journal of the International Neuropsychological Society. 2006;12:816-827.

51. Basso A. How intensive/prolonged should an intensive/prolonged treatment be? Aphasiology. 2005;19:975-984.

52. Marshall RC, Freed DB. The personalized cueing method: from the laboratory to the clinic. American Journal of Speech - Language Pathology. 2006;15:103-111.

53. Olsen E, Donald B, Freed DB, Marshall RC. Generalisation of personalised cueing to enhance word finding in natural settings. Aphasiology. 2012;26:618-631.

54. Cherney L, Kaye R, Lee J, van Vuurenc S. Impact of personal relevance on acquisition and generalization of script training for aphasia: a preliminary analysis. American Journal of Speech-Language Pathology. 2015;24:913-922.

55. Meteyard L, Bose A. What does a cue do? comparing phonological and semantic cues for picture naming in aphasia. Journal of Speech, Language, and Hearing Research. 2018;61:658-674.

56. Coster WJ. Making the best match: selecting outcome measures for clinical trials and outcome studies. American Journal of Occu- 
pational Therapy. 2013;67:162-170.

57. Eriksson K, Forsgren E, Hartelius L, Saldert C. Communication partner training of enrolled nurses working in nursing homes with people with communication disorders caused by stroke or Parkinson's disease. Disability and Rehabilitation. 2016;38:1187-1203.

58. Rautakoski P, Korpijaakko-Huuhka AM, Klippi A. People with severe and moderate aphasia and their partners as estimators of communicative skills: a client-centred evaluation. Aphasiology. 2008;22:1269-1293.

59. Code C. Apportioning time for aphasia rehabilitation. Aphasiology. 2012;26:729-735.

60. Robey RR. A five-phase model for clinical-outcome research. Journal of Communication Disorders. 2004;37:401-411. 